\title{
CREACIÓN DE ATLAS FOTOGRÁFICOS DE BOTÁNICA, FLORA, VEGETACIÓN Y PAISAJES PARA LA MEJORA DEL PROCESO DE ENSEÑANZA-APRENDIZAJE EN ASIGNATURAS DE LOS GRADOS DE BIOLOGÍA Y CIENCIAS AMBIENTALES
}

\author{
CREATION OF PHOTOGRAPHIC ATLAS OF BOTANY, FLORA, VEGETATION \\ AND LANDSCAPES FOR THE IMPROVEMENT OF THE TEACHING-LEARNING \\ PROCESS IN SUBJECTS OF THE DEGREES \\ OF BIOLOGY AND ENVIRONMENTAL SCIENCES
}

http://dx.doi.org/10.15304/ie.29.5668

\author{
Marta Recio Criado \\ martarc@uma.es \\ María Altamirano Jeschke \\ Elena Bañares España \\ Baltasar Cabezudo Artero \\ Blanca Díez Garretas \\ Antonio Flores Moya \\ José García Sánchez \\ Noelia Hidalgo Triana \\ Inmaculada Lozano Torelli \\ Teresa Navarro del Águila \\ Andrés Vicente Pérez Latorre \\ Antonio Picornell Rodríguez \\ Enrique Salvo Tierra \\ $\mathbf{M}^{\mathrm{a}}$ Mar Trigo Pérez \\ Universidad de Málaga ${ }^{1}$
}

\section{RESUMEN}

Se muestran los resultados obtenidos en dos Proyectos de Innovación Educativa de la Universidad de Málaga (PIE10-013 y PIE13-047), desarrollados en dos titulaciones oficiales de grado (Grado de Biología y Grado de Ciencias Ambientales) y en un total de seis asignaturas pertenecientes a la misma área de conocimiento (Botánica). Los principales objetivos fueron la creación de Atlas Virtuales de Prácticas de Botánica, Flora y Vegetación, y su aplicación práctica mediante cuestionarios de

\section{Recibido: 10/XII/2018. Aceptado: X/2019}

M. Recio, M. Altamirano, E. Bañares, B. Cabezudo, B. Díez, A. Flores, N. Hidalgo, I. Lozano, T. Navarro, A. V. Pérez, A. Picornell, E. Salvo y M. M. Trigo, del Departamento de Botánica y Fisiología Vegetal de la Universidad de Málaga. J. García del SCAi (Servicio Central de Apoyo a la Investigación) de la Universidad de Málaga. 
autoevaluación a través del Campus Virtual. Estos atlas están publicados en la web del Departamento de Botánica y Fisiología Vegetal de esta universidad. El Atlas de Prácticas de Botánica consta de 10 carpetas, cada una de ellas correspondientes a diferentes grupos vegetales, y un total de 482 páginas de composición de imágenes fotográficas. Actualmente, el Atlas de Flora cuenta con más de 1000 fotografías, y en él se incluyen índices alfabéticos de taxones (775) y familias (111), cada uno de ellos con su correspondiente enlace a la página de imágenes. Constituye, por tanto, un rápido y práctico buscador de imágenes de plantas, avalado además por profesores universitarios con experiencia, por lo que presenta garantía y calidad científica. El Atlas de Vegetación cuenta con cerca de 500 páginas de imágenes fotográficas, repartidas en cuatro bloques: Bosques (137), Arbustedas y Matorrales (86), Comunidades riparias (67) y Otras comunidades (103). En cada uno de ellos se incluye un índice ordenado y numerado, con objeto de facilitar la búsqueda de determinadas formaciones vegetales, así como de conocer su identidad y clasificación. Se ha creado también Atlas de Paisajes Vegetales y Atlas de Angiospermas-Flores. Todos contribuyen de manera sustancial a mejorar el proceso de enseñanzaaprendizaje en varias asignaturas de los grados de Biología y Ciencias Ambientales. Su uso y aplicación en pruebas de evaluación evita la sobrecarga de trabajo del profesorado.

Palabras clave: web, botánica, fotografías, flora, vegetación.

\section{ABSTRACT}

The results obtained in two Educational Innovation Projects of the University of Malaga (PIE10-013 and PIE13-047) are shown. They have been developed in two official degrees (Biology Degree and Environmental Science Degree) and in a total of six subjects belonging to the same area of knowledge (Botany). The main objectives were the creation of Virtual Atlas of Botany, Flora and Vegetation, and their practical application by questionnaires in the Virtual Campus. These atlas are published on the website of the Department of Botany and Plat Physiology of this university. The Atlas of Botanical Practices consists of 10 folders, each of them corresponding to different plant groups, and a total of 482 pages of composition of photographic images. Currently, the Atlas of Flora has more than 1000 photographs, and it includes alphabetical indexes of taxa (775) and families (111), each with its corresponding link to the image page. It constitutes, therefore, a fast and practical image search engine for plants, also supported by experienced university professors, for which it presents scientific quality. The Atlas of Vegetation has about 500 pages of photographic images, divided into four blocks: Forests (137), Scrub (86), Riparian Communities (67) and Other Communities (103). An ordered and numbered index is included for each, in order to facilitate the search of certain plant communities, as well as to know their identity and classification. Atlas of Landscapes and Angiosperms-Flowers have also created. All contribute substantially to improve the teaching-learning process in several subjects of the degrees of Biology and Environmental Sciences. Its use and application in evaluation tests avoids the overload of work for teachers.

Key words: web, botany, photographs, flora, vegetation.

\section{INTRODUCCIÓN}

Fue en el curso académico 2010-11 cuando, debido a la necesidad de convergencia en el Espacio Europeo de Enseñanza Superior (EEES), se implantaron las titulaciones de grados de Biología y de Ciencias Ambientales en la Facultad de Ciencias de la Universidad de Málaga (UMA), sustituyendo a las antiguas licenciaturas con el mismo nombre. El profesorado se veía obligado a realizar nuevas tareas: actividades formativas y evaluaciones continuas del alumnado para la adquisición de competencias generales del grado y específicas de asignaturas. 
La mayoría de las asignaturas que se imparten en ambos grados se caracterizan por presentar un marcado carácter experimental, por lo que las actividades prácticas juegan un importante papel y constituye un número considerable de horas de trabajo del alumno (créditos presenciales y no presenciales). Los alumnos de primer y segundo curso de ambos grados están realizando prácticas de laboratorio o campo durante prácticamente todas las semanas del curso. En el caso de asignaturas básicas y obligatorias adscritas al área de Botánica, se realizan entre 6 y 11 prácticas por asignatura durante un semestre.

Uno de los principales problemas que se planteaba era el excesivo número de alumnos matriculados en asignaturas básicas y obligatorias (cerca de 200 o más), lo que hace prácticamente imposible el seguimiento individual de cada alumno por parte del profesor en sus actividades formativas. Por ello, se pensó en la posibilidad de evaluar las actividades prácticas y la adquisición de competencias mediante la realización de pruebas de conocimiento a través de la plataforma Moodle del campus virtual de la Universidad de Málaga, ya que permite calificar de manera automática, sin la presencia del profesor.

Otro de los problemas que se nos planteaba era la falta de recursos didácticos para el estudio y aprendizaje de los contenidos que se dan durante las clases prácticas de Botánica. Por ello publicamos un libro denominado Manual y Guión de Prácticas de Botánica (Recio et al. 2008) que, al agotarse su edición, dejamos disponible (en formato PDF) en la página web de nuestro Departamento de Botánica y Fisiología Vegetal (www.biolveg.uma.es/atlas fotografico/inicio.html). Pero ese libro, a pesar de su gran utilidad práctica para entender y asimilar conceptos y estructuras vegetales puesto que incluye una gran cantidad de dibujos ilustrativos, carece de imágenes fotográficas reales. Por ello, se pensó en iniciar este proyecto de creación de unos atlas fotográficos que además estuvieran disponibles en Internet para uso tanto por parte del profesor en el laboratorio durante las prácticas, sirviendo de recurso de apoyo para la docencia práctica, como por parte de los alumnos, para estudio, aprendizaje, refuerzo y repaso del material observado en prácticas de laboratorio o campo.

La finalidad de esta experiencia consiste, por tanto, en la creación y puesta en marcha de recursos digitales fotográficos de calidad científica y didáctica para la docencia y el aprendizaje en el área de Botánica.

\section{OBJETIVOS}

1) Crear un atlas fotográfico de Botánica, de una manera ordenada y clasificada y elaborando láminas de composición fotográfica de las partes vegetativas y reproductoras de los diferentes grupos vegetales: cianobacterias, algas, hongos, líquenes, briófitos, cormófitos (pteridófitos, gimnospermas y angiospermas), que sirva como recurso didáctico de apoyo para la docencia y el aprendizaje de las prácticas de botánica que se imparten en las asignaturas básicas y obligatorias de los grados de Biología y de Ciencias Ambientales.

2) Crear bancos de preguntas de prácticas de botánica, así como de conceptos teóricos básicos y principios fundamentales de botánica general, en el campus virtual de la UMA y aplicarlos mediante actividades de tipo cuestionarios (pruebas de conocimiento) con objeto de: mejorar el rendimiento 
académico del alumno a través del aprendizaje autónomo y la autoevaluación continua, incluir las competencias en las evaluaciones del alumno (competencias generales y específicas), y conseguir un enfoque mixto en las asignaturas del grado de Biología y Ciencias Ambientales pertenecientes al área de Botánica: docencia presencial y docencia virtual, es decir docencia semipresencial (blendedlearning).

3) Crear, de una manera ordenada y clasificada, atlas fotográficos de Flora, de Comunidades Vegetales y de Paisajes Vegetales que sirva de apoyo para la docencia y el aprendizaje en varias asignaturas del área de Botánica, de los grados de Biología y Ciencias Ambientales.

4) Dar un paso más en el uso de los entornos virtuales, no solamente a través de los recursos y la potencialidad de Moodle en el Campus Virtual de la Universidad de Málaga, sino también a través de otras webs docentes, facilitando la búsqueda de fotografías de calidad científica y didáctica de unidades de flora (taxones de angiospermas), vegetación (comunidades vegetales) y paisaje vegetal.

5) Hacer uso de una evaluación continua del alumnado de manera no presencial, eliminando la carga adicional del profesorado en la evaluación continua.

6) Involucrar al alumno a reforzar y reflexionar sobre cada práctica realizada en el laboratorio o campo, y también sobre los contenidos teóricos fundamentales de la asignatura.

7) Intentar lograr una mayor motivación de los alumnos por las asignaturas del área de botánica.

\section{CONTEXTO}

Los proyectos de innovación educativa PIE10-013 y PIE13-047 de la UMA se han desarrollado en dos titulaciones oficiales de grado (Grado de Biología y Grado de Ciencias Ambientales) y un total de seis asignaturas pertenecientes a la misma área de conocimiento (Botánica).

\section{Grado en Ciencias Ambientales:}

\section{Asignatura: Botánica}

Se trata de una asignatura de carácter básico (módulo de Materias Básicas) que se imparte en el Primer Curso en dos grupos grandes de teoría, con un total de unos 150 alumnos. Consta de 9 créditos ECTS (total presencial 90 horas, total no presencial 135 horas). Se impartió por primera vez en la UMA durante el periodo de vigencia del primer proyecto de innovación educativa (curso 2010-11, PIE10-013).

Los profesores implicados en la docencia teórica son 1 ó 2 por grupo grande, mientras que la docencia práctica se implican gran parte de los profesores del área de Botánica del departamento de Botánica y Fisiología Vegetal. Solamente imparte un profesor en cada grupo de práctica, siendo 6 el número de grupos de alumnos de prácticas y 12 el número total de prácticas que se realizan en esta asignatura (una por semana, 11 en el laboratorio y otra en el campo).

El número de alumnos por grupo de práctica gira en torno a los 25 , por lo que la interacción entre alumno-profesor se hace difícil y hemos tenido que auxiliarnos de los recursos docentes que se 
exponen en este proyecto de innovación educativa (atlas fotográfico de prácticas de botánica), para mejorar nuestra calidad docente. Por otro lado, los alumnos los han empleado en su preparación, estudio y aprendizaje constructivista para realizar el examen final práctico. Este atlas ha sido abordado desde que se inició este grado en la UMA, y continúa utilizándose.

\section{Asignatura: Técnicas y Análisis de la Vegetación y Paisaje}

Se trata de una asignatura de carácter optativo (módulo Optatividad) de Cuarto Curso, que se ha impartido por primera vez en la UMA durante el curso académico 2013-14, y continuó durante los siguientes cursos con un total de 11-15 alumnos matriculados. Consta de 6 créditos ECTS (total presencial 60 horas, total no presencial 90 horas).

Los profesores implicados en la docencia teórica y práctica fueron dos, ambos participantes del proyecto. En esta asignatura se realizan 3 prácticas de laboratorio y dos de campo. Los alumnos han hecho uso de los atlas de flora, vegetación y paisaje creados en este proyecto para elaborar sus trabajos de campo (trabajo de curso). También aportaron algunas fotografías para el atlas de vegetación y paisaje.

\section{Grado en Biología:}

\section{Asignatura: Botánica-I}

Se trata de una asignatura de carácter obligatorio (módulo de carácter Fundamental Botánica) que se imparte en el Segundo Curso en dos grupos grandes de teoría, con un total de unos 200 alumnos. Consta de 6 créditos ECTS (total presencial 60 horas, total no presencial 90 horas).

Los profesores implicados en la docencia teórica son 1 ó 2 por grupo, mientras que en la docencia práctica se implica gran parte de los profesores del área de Botánica, normalmente uno por grupo de práctica, habiendo sido 7, 8 ó 9 el número de grupos reducidos de prácticas (de 18-25 alumnos) y 6 el número total de prácticas que se realizan en el laboratorio (una por semana).

Debido al elevado número de alumnos para un solo profesor, al igual que en la anterior asignatura citada (Botánica, del Grado de Ciencias Ambientales), para mejorar la docencia práctica los profesores se han auxiliado de los atlas fotográficos de práctica de botánica, y los alumnos han hecho uso de ello para su preparación, estudio y aprendizaje, así como para prepararse el examen y elaborar los cuadernos de práctica.

\section{Asignatura: Botánica-II}

Esta asignatura tiene las mismas características y créditos que la asignatura anterior. Los alumnos son los mismos, pero se imparte en el segundo semestre. El número de profesores implicados en la docencia teórica y práctica, así como el número de alumnos por grupo de práctica es también similar. El número total de prácticas de laboratorio que se realizan en esta asignatura es también de 6 (una por semana) pero tiene además una práctica de campo y una visita al Jardín Botánico de la UMA. 
También se han hecho esfuerzos enfocados a los mismos objetivos que se ha referido anteriormente. En este caso, al contener la asignatura temas teóricos y prácticos de gimnospermas, angiospermas, flora y vegetación, se ha hecho uso de todos los atlas virtuales creados en este proyecto.

\section{Asignatura: Biodiversidad Vegetal}

Se trata de una asignatura de carácter optativo (módulo de Optatividad) que se imparte en Cuarto Curso. Se impartió por primera vez en la UMA durante el curso académico 2013-14 y continuó durante los siguientes cursos, con un total de 10-36 alumnos matriculados. Consta de 6 créditos ECTS (total presencial 60 horas, total no presencial 90 horas).

Las profesoras implicadas en la docencia teórica han sido dos, siendo ambas participantes del proyecto. Se realizaron dos prácticas de campo y dos prácticas de laboratorio. Los alumnos realizaron trabajos de campo y de curso haciendo uso de los atlas virtuales creados en este proyecto, además de aportar algunas fotografías para los atlas de flora, vegetación y algas. Además, estos atlas han sido muy empleados por los alumnos como buscador y para consulta, facilitando la búsqueda de información de calidad didáctica y el aprendizaje autónomo.

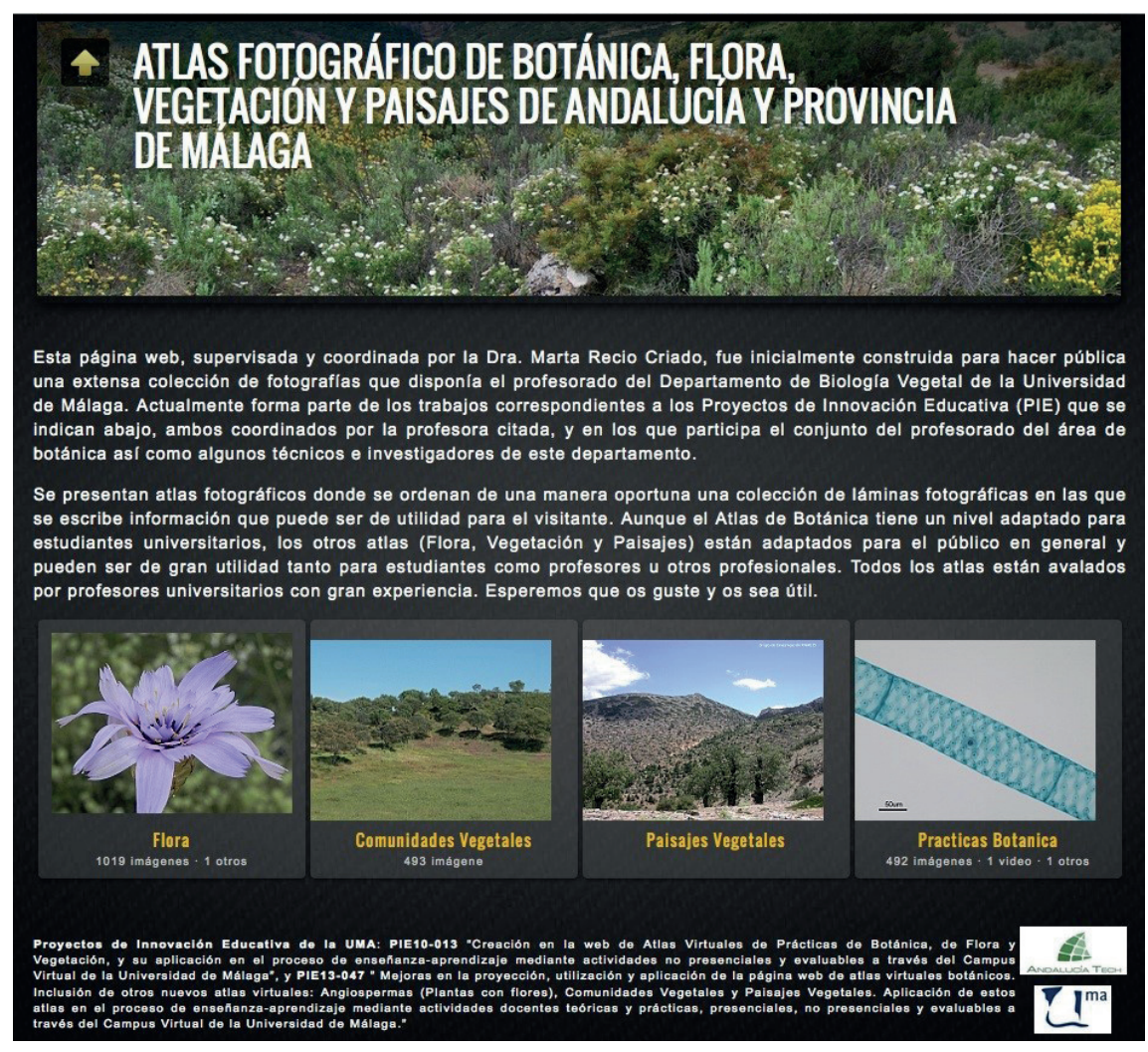

Figura 1. Portal web creado en este proyecto. 


\section{Asignatura: Botánica Ambiental y Aplicada}

Se trata de una asignatura de carácter optativo (módulo de Optatividad) que se imparte en Cuarto Curso. Se impartió por primera vez en la UMA durante el curso académico 2013-14 y continuó durante los siguientes cursos, con un total de 27-40 alumnos matriculados. Consta de 6 créditos ECTS (total presencial 60 horas, total no presencial 90 horas).

La asignatura se impartió con un solo profesor. Los alumnos participaron en este proyecto aportando fotografías de paisaje, vegetación y flora. La mayoría utilizaron los atlas virtuales como fuente de información para elaborar sus trabajos de curso. Además, ha sido muy usado como buscador y para consulta.

\section{DESCRIPCIÓNY METODOLOGÍA DE LA EXPERIENCIA}

En la web del Departamento de Botánica y Fisiología Vegetal (http://www.biolveg.uma.es) se ha creado un "Atlas Fotográfico de Botánica, Flora, Vegetación y Paisajes de Andalucía y Provincia de Málaga” (http://www.biolveg.uma.es/atlas fotografico/inicio.html) (Figura 1).

\section{Experiencia con el Atlas Fotográfico de Prácticas de Botánica}

Este atlas virtual consta de 10 carpetas (Figura 2), cada una correspondiente a una práctica distinta de la asignatura Botánica de los grados de Biología y de Ciencias Ambientales. Dispone de un total de 482 páginas de composición de imágenes fotográficas, de las cuales el 32\% contiene fotografías realizadas a microscopía óptica y un 22\% fotografías realizadas con la lupa (Figura 3). Para la consecución de estas fotografías, se ha utilizado tanto material vegetal fresco, como material vegetal seco, conservado y prensado de herbario, o material vegetal fijado con formol en recipientes o en portas y cubres de cristal. Las fotografías con aumentos se realizaron con fotolupa y fotomicroscopio de los Servicios Centrales de Apoyo a la Investigación (SCAI) de la UMA, indicándose siempre el aumento efectuado. Algunas de las fotografías realizadas con la lupa fueron muy conseguidas con ayuda del programa informático Helicon-Focus, al realizarse superposiciones de varias imágenes iguales pero con diferentes planos de enfoque, para de esta manera conseguir una percepción en 3D y eliminar zonas desenfocadas (Figura 4).

Se ha implementado para la docencia práctica, proyectándose con ayuda de un cañón de vídeo en el panel del laboratorio de prácticas durante la realización de todas las prácticas de laboratorio de la asignatura Botánica-I de $2^{\circ}$ Grado de Biología y 7 de las 11 prácticas que tiene la asignatura Botánica de $1^{\circ}$ Grado de Ciencias Ambientales. 


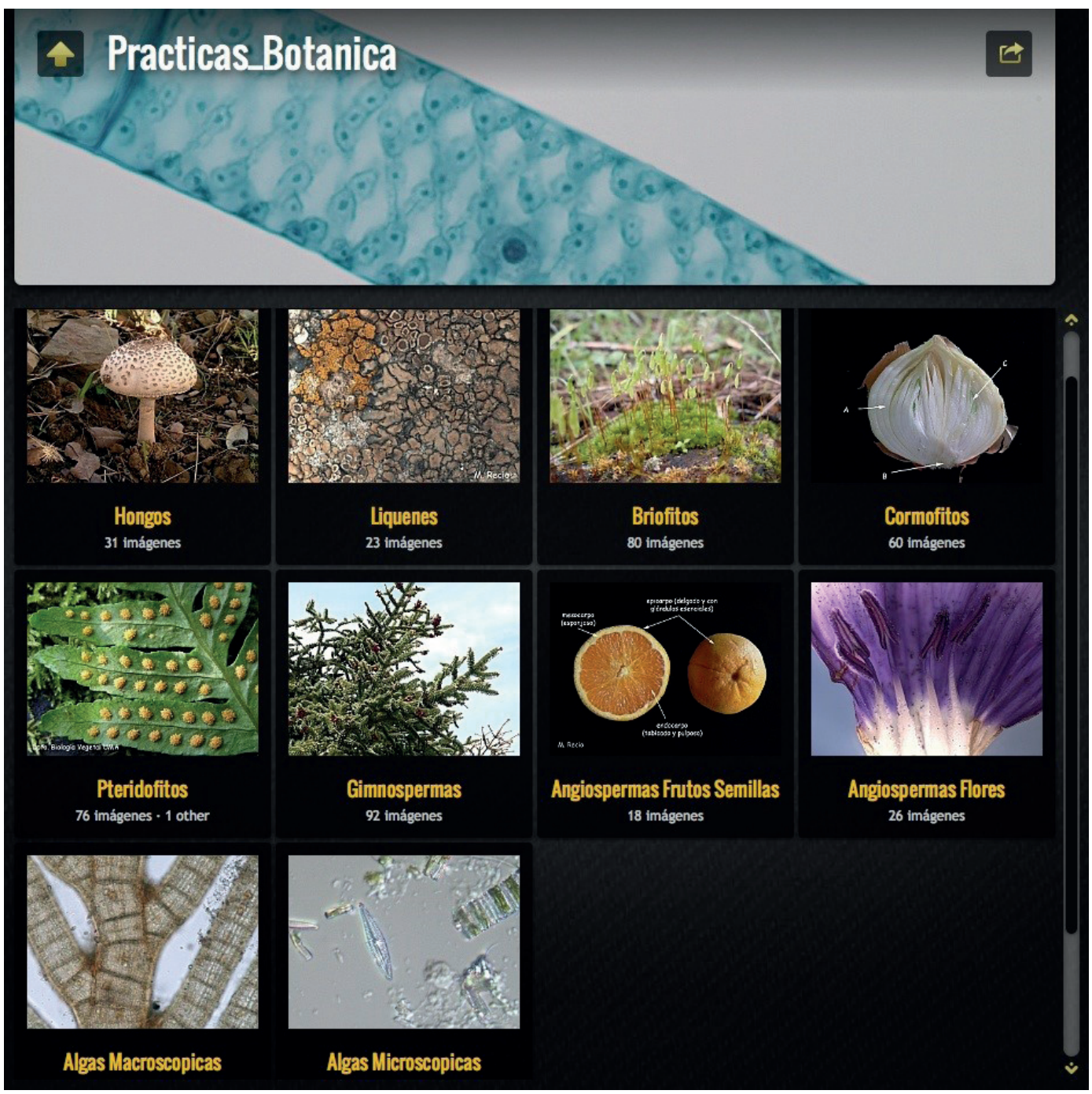

Figura 2. Portal del Atlas Fotográfico de Prácticas de Botánica.

Tras la realización de cada una de las prácticas, este atlas publicado en Internet ha sido empleado asiduamente para estudio y consulta por parte de los alumnos de los dos grados citados anteriormente. Los del grado de Biología lo emplearon puesto que tenían que responder a unos cuestionarios a través del campus virtual (un cuestionario por cada práctica de laboratorio), y los del grado de Ciencias Ambientales porque tenían que realizar un examen final práctico. Ambas tareas realizadas por los alumnos (cuestionarios autoevaluables no presenciales y examen práctico presencial) han sido elementos de evaluación en las correspondientes asignaturas de los grados. 


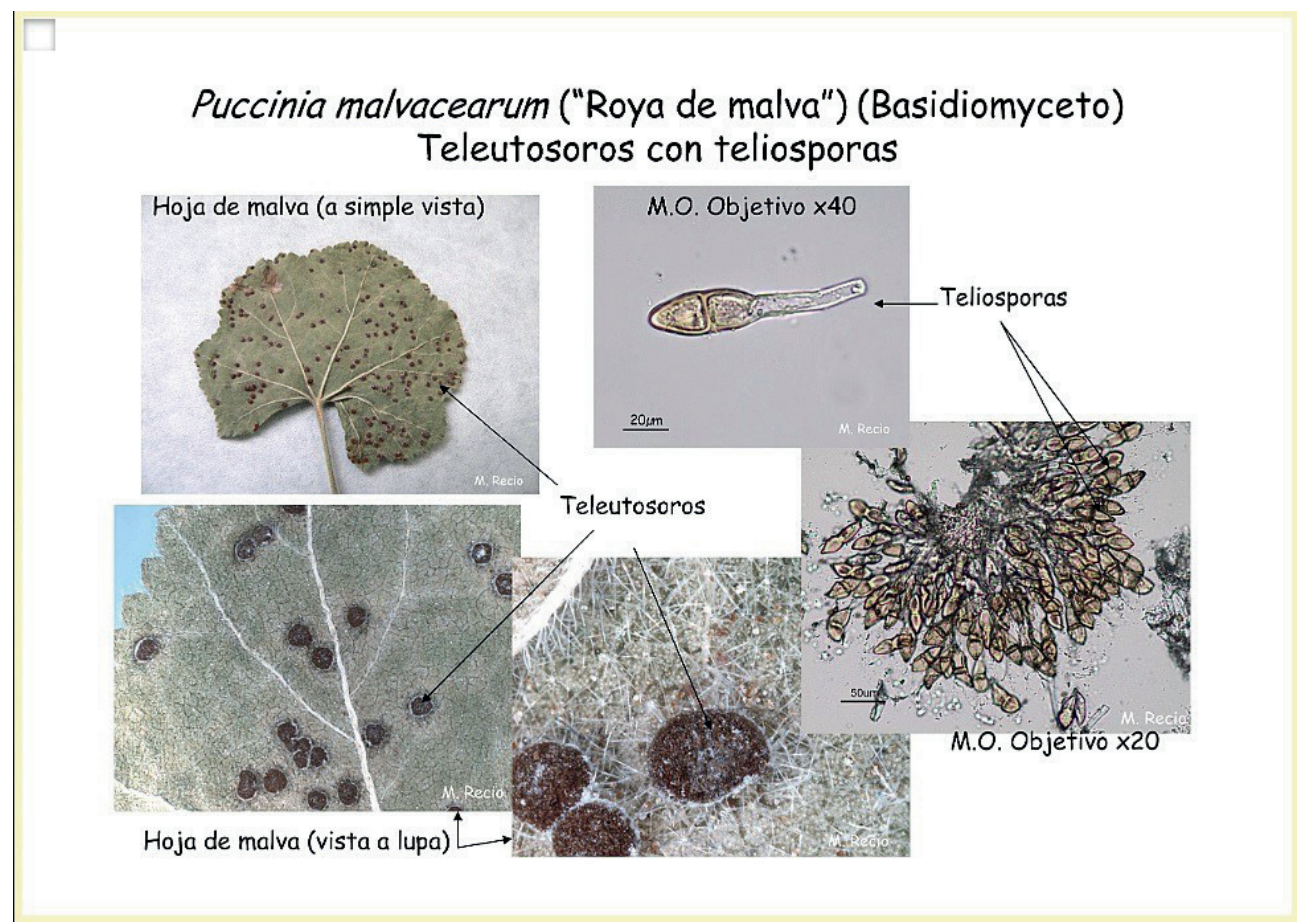

Figura 3. Una de las láminas fotográficas del Atlas de Prácticas de Botánica: Hongos (incluye fotografías realizadas a lupa y microscopía óptica).

Tanto en la Botánica del grado de Biología como en la del grado de Ciencias Ambientales, los alumnos realizan varias prácticas de laboratorio (las 4 últimas) consistentes en la observación a lupa binocular de flores y dibujar fórmulas y diagramas florales de varias especies. El apoyo del profesor al alumno en estas prácticas (con observaciones a lupa) es imprescindible, y como el número de alumnos por grupo es muy elevado, el profesor necesita de algún recurso docente que facilite la enseñanza. Por ello, hemos creado otro atlas fotográfico durante el último curso académico, dentro del Atlas Fotográfico de prácticas de Botánica, el Atlas Fotográfico de Angiospermas-Flores. Durante la primavera, y siempre que hemos tenido hueco libre de docencia, se han recogido flores frescas que se han diseccionado con el auxilio de lupa, agujas y pinzas, y se han realizado fotografías con la lupa del servicio de microscopía del SCAI. En algunos casos se han fotografiado varios planos de la misma imagen floral a lupa y se ha empleado el software Helicon-Focus, que superpone todos los planos fotografiados y selecciona las partes nítidas de cada plano, tratando de conseguir una imagen final más parecida a la real (como lo veríamos con nuestros ojos en la lupa). En otros casos se han fotografiado las flores usando el macro de cámaras fotográficas personales. Todo el proceso necesario, desde que se fotografía hasta que se trasfiere al servidor de la web, ha seguido la misma metodología que los atlas anteriores. Hasta la fecha se han publicado en la web más de 25 láminas de imágenes para las prácticas de Angiospermas. La evaluación de estas prácticas siempre se ha llevado a cabo mediante examen práctico, en el laboratorio. 


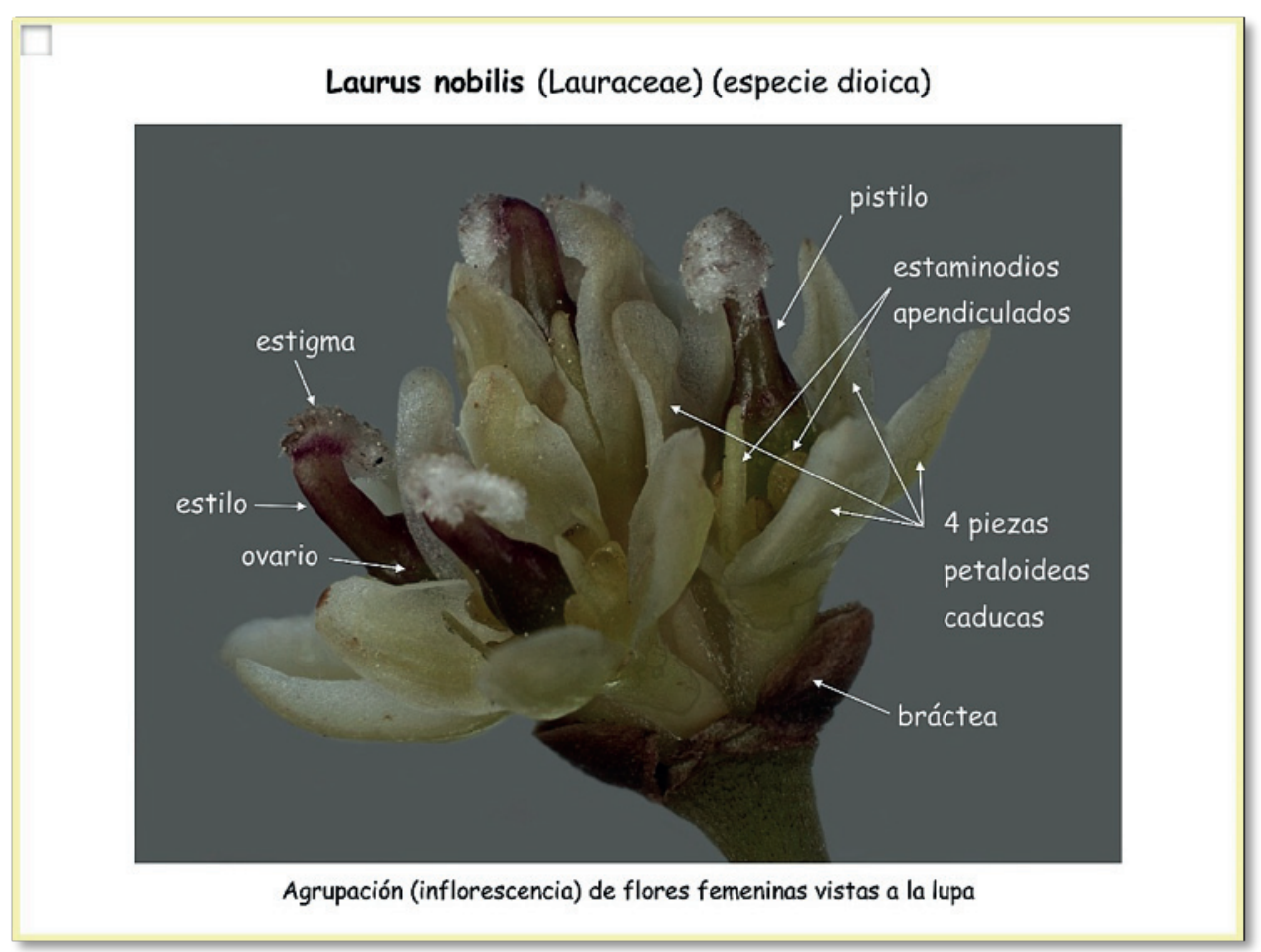

Figura 4. Una de las láminas fotográficas del Atlas de Prácticas de Botánica: Angiospermas-Flores (incluye fotografías realizadas a lupa y uso de Helicon Focus).

\section{Experiencia con el Atlas Fotográfico de Flora}

Para la elaboración de este atlas, se llevaron a cabo, durante los primeros meses del proyecto, actividades consistentes en la creación de un espacio virtual de la UMA donde se incluyó un "Listado de plantas que faltan por hacer fotos". Establecimos así un foro en el que los investigadores participantes del proyecto hemos estado trabajado conjuntamente enviando fotos de flora y discutiendo su identificación. Hemos conseguido completar el atlas de Flora de Angiospermas con más de 1000 páginas de imágenes, repartidas en Monocotiledóneas, Magnólidas y Eudicotiledóneas. Para la flora de Pteridófitos (helechos) se incluyó un archivo PDF con más de 80 imágenes fotográficas (Cabezudo y Trigo, 2004).

Esta Flora Virtual de Andalucía y provincia de Málaga consta de 5 carpetas (Figura 5) donde se ordenan los diferentes grupos taxonómicos de plantas con flores y frutos (Angiospermas) por criterio evolutivo, según como está publicado en la Flora Vascular de Andalucía Oriental (Blanca et al. 2011), que sigue la propuesta de Soltis et al. (2000) y de The Angiosperm Phylogeny Group (2003). En cada carpeta hay una primera página que constituye el índice del contenido de dicha carpeta, numerándose secuencialmente cada nuevo grupo y subgrupo taxonómico (desde la 
categoría superior de Subclase hasta la de Familia, pasando por la intermedias de Superorden y Orden). Después van apareciendo cada uno de los taxones (especies o subespecies) con una o más fotografías que muestran el porte, detalle de la hoja, flor, fruto, etc. También se indica, para cada taxon, el nombre científico, los nombres vulgares, las categorías supraespecíficas a la que pertenece (su numeración en el índice) y las características del hábitat donde vive.

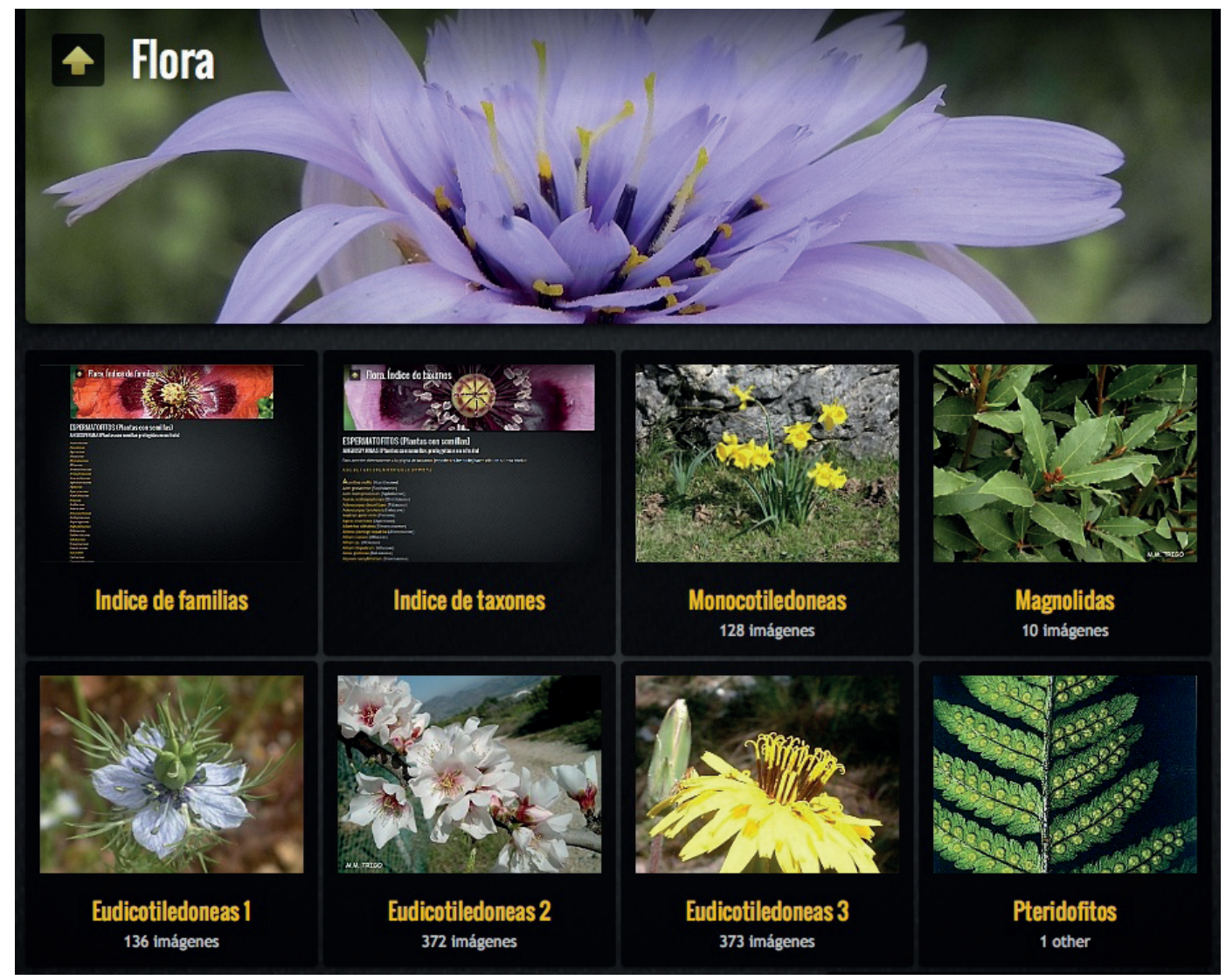

Figura 5. Portal del Atlas Fotográfico de Flora.

En este Atlas de Flora se ha incluido un archivo HTML de índice alfabético de taxones (775 taxones) y de otro de familias (111 familias), en el que se ha realizado el enlace de cada taxon (o familia) a su web de imágenes. De esta manera hemos creado un rápido y práctico buscador, pues esta lista facilita la búsqueda y permite el acceso directo a la lámina del taxon buscado.

\section{Experiencia con el Atlas Fotográfico de Comunidades Vegetales}

Para la elaboración de este atlas hemos realizado una labor importante de identificación sintaxonómica de las formaciones vegetales que se observaban en las fotografías, para lo que hemos 
contado con la ayuda de los participantes expertos en fitosociología. Este atlas se ha completado con cerca de 500 páginas de imágenes fotográficas, repartidas en cuatro bloques: Bosques (137 páginas), Arbustedas y Matorrales (89 páginas), Comunidades riparias (67 páginas) y Otras comunidades (203 páginas) (Figura 6).

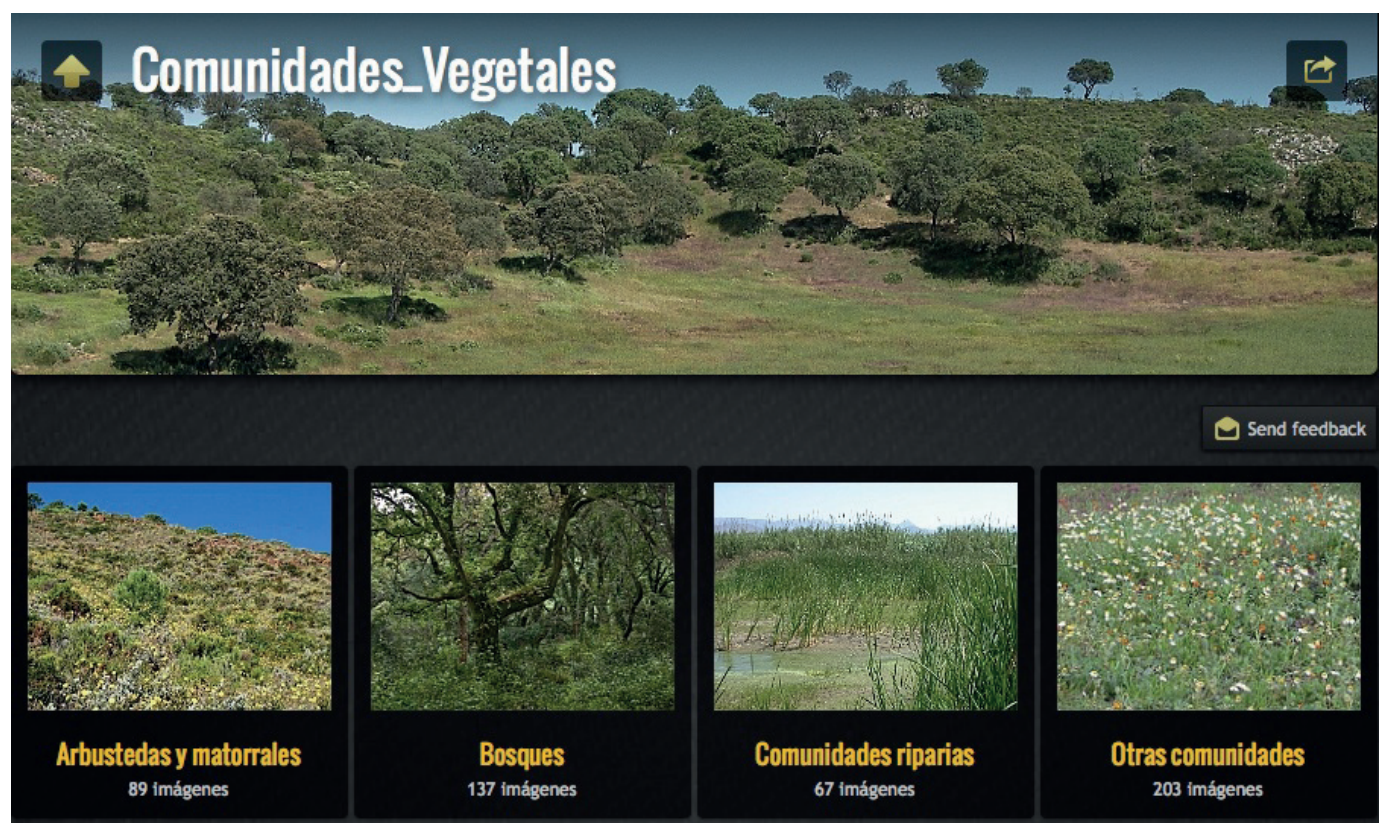

Figura 6. Portal del Atlas Fotográfico de Comunidades Vegetales.

En cada uno de estos bloques inicialmente hay un índice ordenado y numerado de formaciones vegetales, con objeto de facilitar la búsqueda de fotografías de determinadas comunidades vegetales. Cada página (siempre ordenada y numerada según el índice) presenta una o varias imágenes fotográficas que contemplan las características de una comunidad vegetal determinada, además de estar acompañada de un título y algunas indicaciones importantes. El usuario puede ir pasando las páginas al tiempo que va leyendo su contenido y su numeración, informándose de esta manera de las diferencias que se van observando entre las distintas comunidades de un mismo grupo. Por tanto hay que destacar que nuestro portal web no es un simple álbum de fotos, sino un atlas científico ordenado de comunidades vegetales, que aporta información muy valiosa tanto desde el punto de vista educativo como científico. 


\section{Experiencia con el Atlas Fotográfico de Paisajes Vegetales}

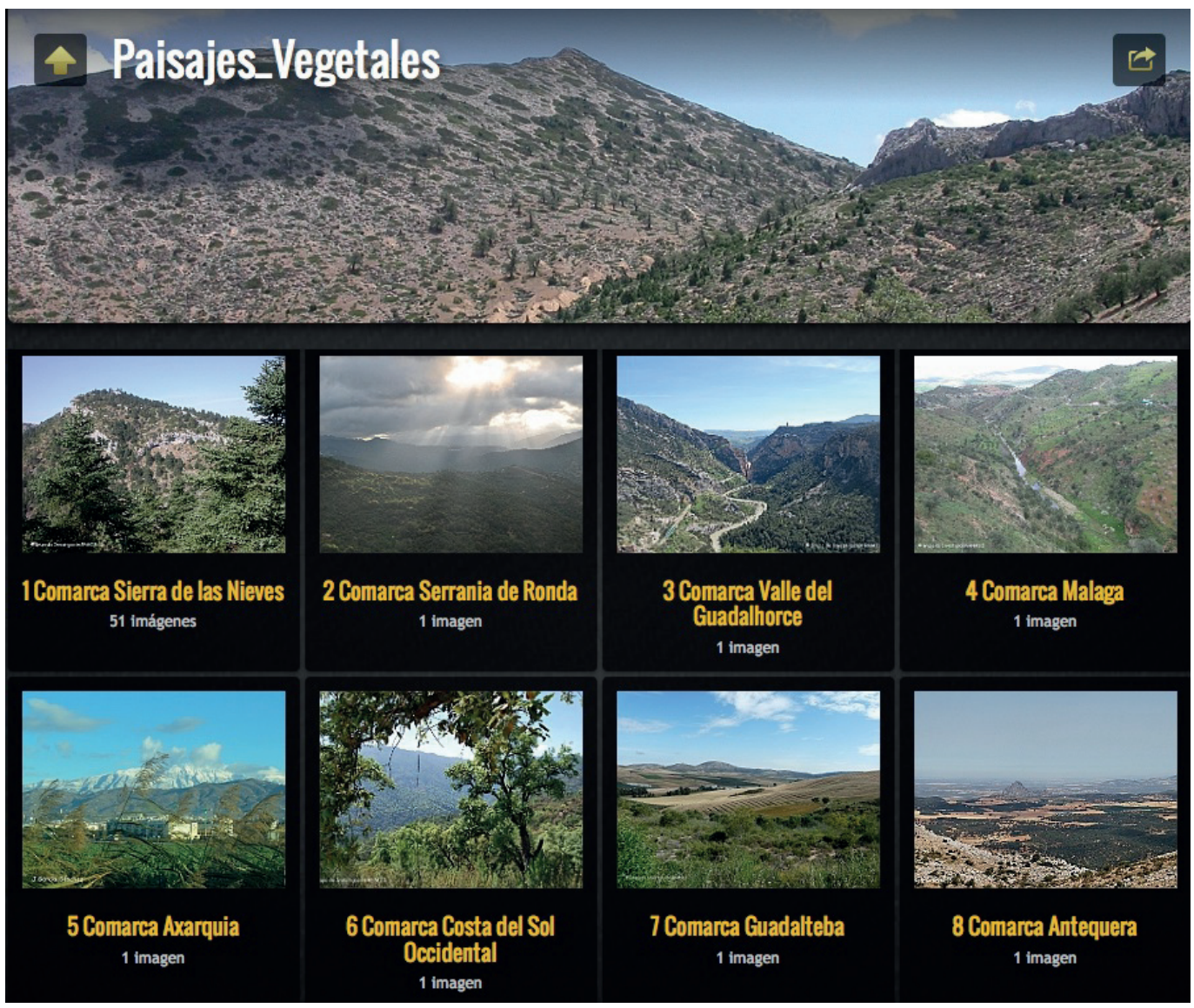

Figura 7. Portal del Atlas Fotográfico de Paisajes Vegetales.

Respecto al Atlas de Paisajes Vegetales (Figura 7), se ha estado trabajando en la selección y clasificación (ordenación) de fotografías que todo el equipo participante iba suministrando a la coordinadora del proyecto. Se han elaborado tantas carpetas como comarcas de la provincia de Málaga, y dentro de cada una de ellas varias carpetas correspondientes a los distintos lugares geográficos de cada comarca. Los diferentes archivos fotográficos recopilados (formato JPG) fueron nombrados, ordenados y guardados en sus correspondientes carpetas. Después se elaboraron las definitivas páginas del atlas (imágenes con texto incluido) en archivos de Power-Point, éstos se pasaron a formato JPG de nuevo, numerando y nombrando cada uno de estos archivos JPG con palabras claves para ser encontrados por buscadores de Internet. Después se crearon los archivos HTML en los programas J-Album y Dreamweaver, y finalmente fueron transferidos al servidor web mediante Filezilla. Todo este procedimiento es el mismo que se hizo con todos los atlas fotográficos citados anteriormente. 


\section{Experiencia con los Cuestionarios de Prácticas de Botánica en el Campus Virtual de la UMA}

Se han elaborado, en el campus virtual de la UMA, un banco de preguntas para prácticas de Botánica que se imparten en diferentes asignaturas y grados (Botánica-I y Botánica-II del grado de Biología, y Botánica del grado de Ciencias Ambientales). Este repositorio contiene preguntas de tantas categorías como prácticas diferentes se realizan en Botánica. Y, dentro de cada categoría, se han hecho subcategorías de preguntas en función de los diferentes objetivos, grupos de organismos vegetales, o tipos de estructuras vegetales que deben observar los alumnos en cada una de las prácticas de botánica. Hasta el momento se han elaborado un total de 302 preguntas de prácticas (la mayoría con imágenes del atlas fotográfico de prácticas citado anteriormente, y de tipo opción múltiple y emparejamiento), repartidas en 10 categorías (correspondientes a 10 prácticas diferentes) y más de 60 subcategorías.

Los alumnos de estas asignaturas realizaron pruebas de evaluación continua de prácticas mediante cuestionarios de autoevaluación en el Campus Virtual de la UMA (pruebas de conocimiento). Tras la realización de la primera práctica de laboratorio, cada semana se abrió en el Campus Virtual pruebas de conocimiento de cada una de las prácticas realizadas en el laboratorio. Estas pruebas fueron obligatorias, no presenciales, de duración limitada y con un solo intento, habiéndose barajado las preguntas al azar para cada alumno. Los tipos de preguntas fueron fundamentalmente de opción múltiple con una o varias respuestas, de emparejamiento y de respuesta corta, la mayoría de ellas con imágenes fotográficas. También se realizaron pruebas de identificación visual de flora, que fueron de carácter obligatorio pero presenciales. Se realizaron en el aula mediante proyección de imágenes fotográficas. Previamente a dicha prueba, los alumnos tuvieron tiempo para el estudio, memorización, reforzamiento y aprendizaje autónomo del Atlas de Flora disponible en la web de nuestro departamento.

A la hora de seleccionar las preguntas y montar el cuestionario para los alumnos, siempre hubo consenso entre los profesores, debido a la larga experiencia que tenemos en la docencia de prácticas de Botánica y a que el tipo de material vegetal que se muestra a los alumnos es muy similar en todos los años.

\section{Experiencia con los Cuestionarios Teóricos de Botánica en el Campus Virtual de la UMA}

Puesto que otros de los objetivos que se plantearon en este proyecto fueron el de involucrar al alumno a reforzar y reflexionar sobre los contenidos teóricos fundamentales de la asignatura, e intentar lograr una mayor motivación de los alumnos por las asignaturas del área de Botánica, pensamos que los cuestionarios autoevaluables del campus virtual son también un buen recurso para lograrlo.

Cada vez que el profesor terminaba de explicar en clase un bloque de temas, se abría en el campus virtual de la asignatura un cuestionario de teoría para ese bloque temático, con el objeto de que los alumnos lo pudieran trabajar libremente cuando quisieran y todas las veces que quisieran para 
su estudio y refuerzo en el aprendizaje. En el campus virtual se elaboró un repositorio con un total de 130 preguntas teóricas, repartidas en 5 categorías, correspondientes a cada uno de los 5 bloques temáticos que contiene el programa teórico de la asignatura en la que se aplicó esta experiencia. Se formularon preguntas que implicaran una reflexión de los conceptos y principios básicos de la asignatura y que el profesor previamente había explicado en clase. A partir de ese repositorio, los profesores de teoría elaboraron 5 cuestionarios de teoría evaluables automáticamente, uno para cada bloque teórico y con 5 a 12 preguntas por cuestionario, a libre disposición para uso y estudio por parte de los alumnos. Se les avisaba a los alumnos que, para la evaluación continua de la asignatura, iban a realizar en el aula dos pruebas parciales de contenidos teóricos, que serían de tipo test y también con preguntas de respuesta corta, en las que se incluirían preguntas de los cuestionarios que el profesor había dejado disponible en campus virtual. Se desconocían las respuestas correctas, obligando al alumno repetir el cuestionario para conocer las respuestas correctas y aprender de los errores cometidos.

Esta experiencia se ha llevado a cabo en la asignatura Botánica-I de $2^{\circ}$ curso del grado de Biología durante el curso 2011-12, con un total de 157 alumnos matriculados.

\section{Encuesta final de valoración de las experiencias}

Debido a que todas las experiencias propuestas se han llevado a cabo plenamente con los alumnos de los grupos A y B de $2^{\circ}$ curso del Grado de Biología matriculados durante el curso 201112 en la asignatura Botánica I, dicha encuesta las han realizado exclusivamente estos alumnos. Concretamente se llevó a cabo, en el aula B1 de la Facultad de Ciencias durante el último día de clase del segundo semestre del curso 2011-12. El número total de alumnos encuestados ha sido de 83, igualmente repartidos en los dos grupos (41 del grupo A y 42 del grupo B). La encuesta tenía un total de 27 preguntas, todas ellas formuladas con objeto de obtener indicadores de calidad del proceso de enseñanza-aprendizaje mediante la utilización de los recursos virtuales que se crearon.

\section{RESULTADOS Y DISCUSIÓN DE LAS EXPERIENCIAS}

Los principales resultados obtenidos han sido la creación de la web "Atlas Fotográfíco de Botánica, Flora, Vegetación y Paisajes de Andalucía y Provincia de Málaga” (http://www.biolveg. uma.es/atlas fotografico/inicio.html) y la creación de los bancos de preguntas y de cuestionarios de autoevaluación (o pruebas de conocimiento) en el Campus Virtual de la UMA para varias asignaturas del área de Botánica. En la mayoría de las preguntas y cuestionarios se incluyen fotos procedentes de esta web.

Para discutir los resultados obtenidos con cada experiencia realizada durante este proyecto, exponemos con detalle los resultados de unas encuestas que se realizaron a los alumnos.

Para recoger las impresiones que habían tenido los alumnos respecto al Atlas Fotográfico de Prácticas de Botánica, se realizaron las 4 preguntas. Se concluye que el 95\% de los alumnos encuestados habían visitado (siempre o casi siempre, antes de responder a cada práctica del 
cuestionario) ese atlas publicado en la web del Departamento de Botánica y Fisiología Vegetal de la UMA. Al 83\% le ha parecido un portal web estupendo y muy útil para repasar y aprender la asignatura. El 78\% creen que ese atlas virtual es útil (señalando mucho) para una mejor comprensión de los contenidos teóricos y prácticos de la asignatura. Además, al 84\% le ha sido útil para responder a las preguntas de los cuestionarios.

Respecto a los Cuestionarios de Prácticas de Botánica, teniendo en cuenta que todos los alumnos encuestados han realizado todas las prácticas de laboratorio y que todos, excepto 9 alumnos del grupo A, han contestado a todos los cuestionarios de prácticas en el campus virtual, el 82\% de ellos están conformes con la calificación obtenida en dichos cuestionarios, que fue buena (una media de notable para el total de 156 alumnos matriculados). El 85\% cree que es importante leerse y repasarse el contenido (libro de prácticas y apuntes de clase) antes de realizar cada práctica en el laboratorio, para aprender y asimilar mejor esos contenidos. Pero afirman que eso lo hizo siempre o casi siempre el $45 \%$, a veces o pocas veces el 52\% y ninguna vez el 7\%. Eso implica la importancia de haber hecho caso a las recomendaciones que se les hizo a través del foro del campus virtual. Se les recomendaba que, siempre y antes de responder a los cuestionarios, leyesen y mirasen el libro de prácticas y el atlas virtual de prácticas, ya que podían hacer libre uso de ello para responder a las preguntas y tenían un tiempo limitado de 20 minutos para responder a todas las preguntas.

Un hecho importante que se detectó en estas encuestas es que la mayoría de los alumnos ( $85 \%$ de los encuestados) afirmaban que había una diferencia importante en la docencia práctica impartida por los diferentes profesores de prácticas de la asignatura, que no se recibía lo mismo con diferentes profesores. En el grupo A impartían dos profesores de prácticas (uno impartía en dos grupos de alumnos, y otro segundo profesor en el tercer grupo de alumnos); y en el grupo B impartían prácticas 3 profesores distintos, uno por grupo. Hubo una diferencia importante en la respuesta a una pregunta de la encuesta, que variaba en función del grupo al que pertenecía el alumno. Casi el $60 \%$ de los alumnos del grupo A (con dos profesores de prácticas) afirmaban que esa diferencia de profesorado les había repercutido en la calificación que obtuvieron en los cuestionarios, mientras que en el grupo B (con tres profesores de práctica diferentes) el 81\% afirmaron que no les había repercutido la diferencia de profesorado, y que la calificación obtenida dependía más del alumno que del profesor. Para evitar ese tipo de problemas, es muy importante que todos los profesores de prácticas estén previamente informados sobre las preguntas que se les va a hacer a los alumnos en los cuestionarios del campus virtual.

A pesar de lo anterior, la metodología realizada en este proyecto para la evaluación de las prácticas, mediante cuestionarios en campus virtual, ha sido adecuada según consta en una pregunta de la encuesta. Se les preguntó a los alumnos cuál método consideraban más adecuado para realizar dichos cuestionarios: justo después de cada práctica si el laboratorio dispusiera de suficientes ordenadores, en un aula de informática todos los alumnos juntos, o como se ha venido haciendo (en casa o donde desee el alumno, tras unos días de haber realizado la práctica). El 87\% respondieron la tercera opción, es decir consideraron que el mejor método era como se hizo.

La metodología de evaluación continua realizada en la asignatura Botánica I de $2^{\circ}$ Grado de Biología ha sido considerada adecuada por casi todos los alumnos encuestados (93\%). Se tuvo en 
cuenta la asistencia a prácticas, los cuestionarios de prácticas realizados en el campus virtual y los exámenes parciales teóricos escritos en los que se incluyeron preguntas tipo test de los cuestionarios teóricos disponibles en campus virtual.

En cuanto a los cuestionarios teóricos, que elaboramos para reforzar el estudio y aprendizaje de los conceptos y principios básicos de la asignatura y que dejamos disponibles en el campus virtual para practicarlo cuantas veces quisieran los alumnos, teniendo en cuenta que se trataba de una actividad voluntaria, ha habido un elevado porcentaje de participación $(60 \%$ de los alumnos matriculados). Además, los alumnos obtuvieron una buena calificación, pues la nota media registrada ha sido 7,3. Los alumnos realizaron varios intentos para responder a los cuestionarios, habiéndose registrado una media de 2,5 intentos/alumno. Las impresiones que han tenido los alumnos de esta experiencia se reflejan en las respuestas a las encuestas que se les pasó al final del semestre: el 88\% de los encuestados confirman que han aprendido con ello (según un $42 \%$ mucho). Hay que tener en cuenta que sólo el $42 \%$ de los encuestados ha hecho todos los cuestionarios teóricos, el 10\% no lo ha hecho, y el $48 \%$ restante no ha hecho todos los que había disponibles (uno por bloque temático). Por tanto, podemos decir que los que hicieron todos los cuestionarios afirman que han aprendido mucho con ellos.

Respecto al Atlas Fotográfico de Flora, la identificación de la flora constituye uno de los bloques teóricos y prácticos más importantes de la asignatura Botánica II de $2^{\circ}$ Grado de Biología y de Botánica de $1^{\circ}$ Grado de Ciencias Ambientales, por lo que es importante que sea evaluado por los alumnos. En lugar de conseguir dicha evaluación a través de un cuestionario en campus virtual, se hizo de una forma más simple: mediante un pequeño examen de proyección Power-Point de imágenes, en la misma aula donde el profesor impartía las clases teóricas. Para el estudio consistente en la identificación y aprendizaje de los nombres de las plantas con flores, los alumnos disponían del Atlas de Flora creado en este proyecto y publicado en la web del Departamento de Botánica y Fisiología Vegetal.

El estudio y reconocimiento de las diferentes comunidades vegetales constituye otro de los grandes bloques teóricos de los programas de las dos asignaturas y grados citados en el párrafo anterior. Para ello se ha creado y publicado en este proyecto el Atlas Fotográfico de Comunidades Vegetales, que ha sido útil para el profesorado del área de Botánica para su docencia, y también para el alumnado (sobre todo de optativas del área), que lo ha empleado fundamentalmente para elaborar trabajos de curso.

\section{CONCLUSIONES}

1) Todos los atlas publicados en la web del Departamento de Botánica y Fisiología Vegetal de la Universidad de Málaga han sido un recurso muy bien valorado por su utilidad, garantía y calidad científica, tanto por los alumnos como por el profesorado universitario de esta universidad y de otras universidades españolas e iberoamericanas.

2) Gracias a los bancos de preguntas y a la metodología de evaluación que propusimos se ha mejorado la calidad del proceso de enseñanza-aprendizaje en las prácticas de botánica. Se trata 
de un proceso de aprendizaje que no necesita de la presencia del profesor. Los cuestionarios de la plataforma Moodle no solamente son una buena técnica de evaluación sino también una buena técnica de aprendizaje para los alumnos, si el profesor hace buen uso de sus recursos.

3) La experiencia con los cuestionarios de prácticas de botánica ha sido muy satisfactoria tanto para los profesores como para los alumnos. Ya no ha sido necesario que los profesores de prácticas preparen exámenes de prácticas y corrijan cuadernos de prácticas. Los profesores más veteranos y que no hacían uso del campus virtual para sus asignaturas han sido convencidos y están satisfechos. Estas evaluaciones automáticas de las prácticas constituyen ser elementos para la evaluación continua, que son necesarios en las asignaturas de los Grados, para su adaptación al Espacio Europeo de Educación Superior (EEES). Además, de esta manera el profesor hace uso de las TIC y, por tanto, cumple con otro de los requisitos del EEES.

4) Se ha conseguido un alto porcentaje de alumnos aprobados y una nota media de notable en las pruebas de evaluación continua de las diferentes prácticas de Botánica usando los bancos de preguntas del Campus Virtual, así como en las pruebas de identificación de angiospermas mediante fotografías. Esto ha ocasionado una mayor motivación por parte de los alumnos a tener interés por las asignaturas del área de Botánica.

5) Los cuestionarios teóricos diseñados para reflexionar y repasar lo que el profesor ha explicado en clases magistrales de teoría son una excelente herramienta de aprendizaje. Con ello los alumnos detectan errores conceptuales $y$, en muchos casos, sirven para corregir errores que detectan en sus apuntes.

\section{REFERENCIAS BIBLIOGRÁFICAS}

Blanca, G., Cabezudo, B., Cueto, M., López C.F. y Morales Torres, C. (eds.) (2011). Flora Vascular de Andalucía Oriental ( $2^{\text {a }}$ edición corregida y aumentada). Universidades de Granada, Almería, Jaén y Málaga.

Cabezudo, B. y Trigo, M. M. (2004). Pteridófitos (helechos y plantas afines). En: G. Blanca-López y A. Ortega-Díaz (Coords.), Proyecto Andalucía, Naturaleza vol. XXI, Botánica II (pp. $315-$ 374). Sevilla: Hércules.

Recio, M. (Coord.), Altamirano Yeschke, M., Asensi Marfil, A., Cabezudo, B., Díez Garretas, B., Flores Moya, A., Navarro del Águila, T., Nieto Caldera, J. M., Pérez Latorre, A. V., Toro Gil, F. J. y Trigo Pérez, M. M. (2008). Manual y Guión de Prácticas de Botánica. Elementos Auxiliares del Clase, (81). Servicio de Publicaciones e Intercambio Científico de la Universidad de Málaga (SPICUM).

Soltis, D.E. et al. (2000). Angiosperm phylogeny inferid from $18 \mathrm{SrDNA}$, rbcL and atpB sequences. Botanical Journal of Linnean Society, 133(4), 381-461. DOI: https://doi.org/10.1006/ bojl.2000.0380

The Angiosperm Phylogeny Group (2003). An update of the Angiosperm Phylogeny Group classifications for the orders and families of flowering plants: APG II. Botanical Journal of Linnean Society, 141(4), 399-436. DOI: https://doi.org/10.1046/i.1095-8339.2003.t01-1-00158.x 